\title{
Wrong vertex displacements due to Lee-Wick resonances at LHC
}

\section{Ezequiel Álvarez*}

Departamento de Física (Universidad de Buenos Aires), CONICET \& IFIBA

E-mail: sequildf.uba.ar

\section{Leandro Da Rold}

CONICET \& Centro Atómico Bariloche, CNEA

E-mail: daroldl@cab.cnea.gov.ar

\section{Carlos Schat}

Departamento de Física (Universidad de Buenos Aires), CONICET, IFIBA \& Department of

Physics and Astronomy (Ohio University)

E-mail: schatedf.uba.ar

\section{Alejandro Szynkman}

Physique des Particules (Université de Montréal)

E-mail: szynkman@lps.umontreal.ca

\begin{abstract}
We show how a resonance from the recently proposed Lee-Wick Standard Model could lead to the observation at the LHC of a here-proposed new observable called wrong vertex displacement. We study which could be the possible 'longest-lived' Lee-Wick particle that could be created at LHC, and we study its possible decays and detections. We conclude through a Monte Carlo simulation that this unique signature for acausal resonances, when analyzed within the Lee-Wick Standard Model, would be measurable in the LHC era if Minimal Flavour Violation is accomplished and if the Lee-Wick electrons mass scale is below $\sim 450 \mathrm{GeV}$.
\end{abstract}

European Physical Society Europhysics Conference on High Energy Physics, EPS-HEP 2009, July 16 - 222009

Krakow, Poland

\footnotetext{
${ }^{*}$ Speaker.
} 
The Lee-Wick Standard Model (LWSM) [1] is a recently proposed extension to the Standard Model which solves the hierarchy problem at the price of introducing a Lee-Wick (LW) partner for each SM particle. The LW particles have the same statistic as their SM partners, but their kinetic term have the opposite sign, leading to partial cancellations in loop corrections that eliminates quadratic divergences. See Ref.[1b] and references therein for a recent review on the LWSM and its consequences.

A very interesting and distinctive feature of the LWSM is its microscopic acausal behaviour, which is originated in the opposite sign in the kinetic terms. Although the theory to be consistent should forbid the temporal exploration of this acausal behaviour, we propose here a new observable called wrong vertex displacement [2] which would explore spatially this acausal behaviour through the creation of a boosted LW resonance.

A wrong vertex displacement is defined as a vertex displacement in which the total momentum of the particles coming from the secondary vertex points towards the primary vertex instead of away of it, and its invariant mass corresponds to a new resonance. It can be shown that this characteristic behaviour corresponds to an acausal resonance [2] and, as expected, the higher is the momentum transferred through the resonance, the higher is the spatial effect due to acausality. On the other hand, it is interesting to notice that the greatest temporal acausal behaviour in the primary vertex occurs in the system of reference where the LW resonance has zero momentum.

In order to find which are the LW particles which would produce the greatest wrong vertex displacements at the LHC, we need to find which have the smaller width, $\Gamma$, or greater 'life-time'. Having into account the LWSM interactions and the constrains on the LW quarks and gauge bosons partners ( $\tilde{W}^{3}$ and $\tilde{B}$ ) coming from the EWPT [3] and those on the LW Higgs [4] we conclude that the LW leptons are the best candidates to produce wrong vertex displacements. Assuming Minimal Flavour Violation (MFV) we obtain, after the proper diagonalization of the Yukawa terms, that the physical states partners of the SM left electron, $\tilde{\ell}^{e}$, and the SM right electron, $\tilde{e}$, are by far the best candidates to produce the sought signature. We plot in Fig.1a the partial and total width of $\tilde{\ell}^{e}$. Since the LWSM favors pair-creation of LW leptons, the characteristic signature at the LHC would be two wrong vertex displacements and it would look like in Fig.1b.

In the following we analyze the experimental perspectives to measure wrong vertex displacements due to a LW electron $\tilde{\ell}^{e}$ at the LHC. In order to observe the sought signal the LW electron needs to be created with the higher possible momentum. Having into account that the LHC detectors have better resolution in the transverse plane we focus on requirements on the transverse momentum $p_{T}$ of the LW electron. If we take as a reference value for the transverse resolution of the secondary vertex position $\Delta x=20 \mu \mathrm{m}$, we find that LW electrons $\tilde{\ell}^{e}$ created with $p_{T}>M_{\ell} \Gamma \Delta x$ will produce a detectable displacement. This allows us to obtain minimum transverse momentum $p_{T}$ for the LW resonance as a function of its mass. For LW masses $M_{\ell}=300,400$ and $500 \mathrm{GeV}$, we obtain that a cut in the transverse momentum of the LW electrons $p_{T}>450,980$ and $1700 \mathrm{GeV}$, respectively, is required to obtain displacements greater than $\Delta x=20 \mu \mathrm{m}$.

We used MadGraph/MadEvent [5] to obtain the total production cross section of a $\overline{\tilde{\ell}}^{e} \tilde{\ell}^{e}$ pair at the LHC with a center of mass energy of $14 \mathrm{TeV}$. We have also computed the cross-section after the above-mentioned cut in $p_{T}$ such that the transverse vertex displacement is greater than $\Delta x=20 \mu \mathrm{m}$. Fig. 2a shows a simulation for the $p_{T}$ distribution and its cut in $\overline{\tilde{\ell}^{e}} \tilde{\ell}^{e}$ production at the LHC. We plot our results for a relevant range of LW masses in Fig. $2 b$, where we have taken $m_{h}=200 \mathrm{GeV}$. 


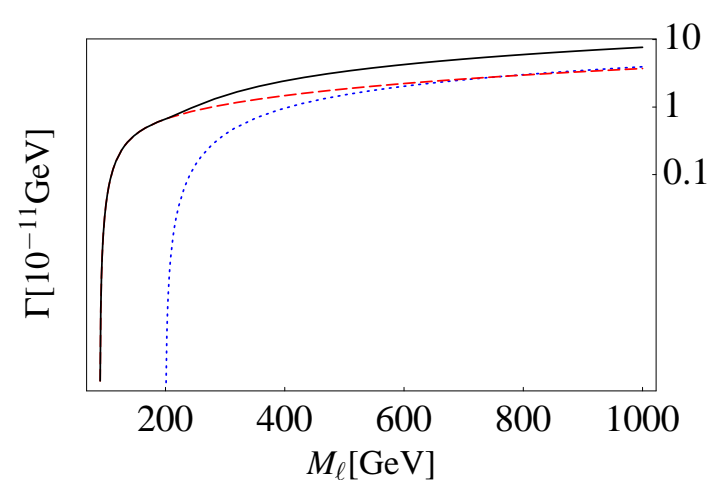

(a)

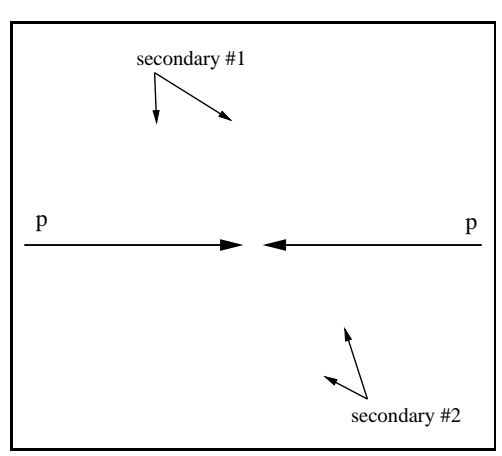

$(b)$

Figure 1: (a) Partial widths of $\tilde{\ell}^{e}$ as a function of its mass for $m_{h}=200 \mathrm{GeV}$. The dotted line corresponds to $\Gamma\left[\tilde{\ell}_{e}^{e} \rightarrow h+e\right]$, the dashed line to $\Gamma\left[\tilde{\ell}^{e} \rightarrow Z+e\right]$ and the solid line to the total width. The neutral current decay dominates the width for $M_{\ell} \lesssim 700 \mathrm{GeV}$ if $m_{h} \gtrsim 200 \mathrm{GeV}$. (b) Two wrong vertex displacement: decay products travel from the secondary towards the primary vertex, instead of moving away from it.

As a general feature we see that the cross section after the cut has a strong dependence with the LW scale. For $M_{\ell}=300 \mathrm{GeV}$ the total cross section is rather large, $\sim 66 \mathrm{fb}$, and after the cut we still have a sensible cross section $\sim 11 \mathrm{fb}$ for $m_{h}=200 \mathrm{GeV}$ and $\sim 8 \mathrm{fb}$ for $m_{h}=150 \mathrm{GeV}$. On the other hand, for $M_{\ell} \gtrsim 500 \mathrm{GeV}$, there is not enough energy at the LHC to create the highly boosted LW leptons.

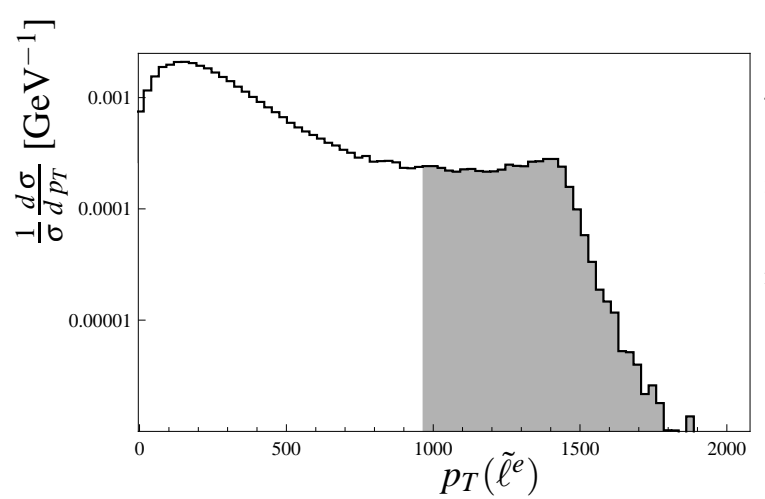

(a)

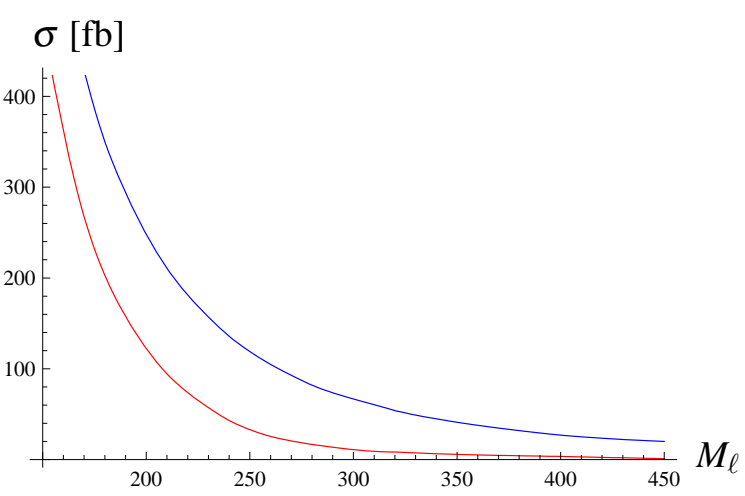

(b)

Figure 2: (a) $p_{T}$ distribution for $\overline{\tilde{\ell}}^{e} \tilde{\ell}^{e}$ production at the LHC setting $M_{\ell}=400 \mathrm{GeV}$. The shaded region -which survives after the cut in $p_{T}$-represents the events that would produce wrong vertex displacements greater than $\Delta x=20 \mu \mathrm{m}$. We used $M_{\tilde{W}}=M_{\tilde{B}}=3 \mathrm{TeV}$ and a center of mass energy of $14 \mathrm{TeV}$. The first resonance corresponds to $Z$ and the second one to $\tilde{W}^{3}$ and $\tilde{B}$, whereas the tail for high $p_{T}$ is due to the lack of available energy in the quarks of the proton's beam. (b) Total (upper line) and $p_{T}$-cut (lower line) cross-sections for $\overline{\tilde{\ell}}^{e} \tilde{\ell}^{e}$ production in LHC. The $p_{T}$ cut ensures that the wrong vertex displacement is greater than $\Delta x=20 \mu m$. In both figures the $x$-axis is in $\mathrm{GeV}$ units.

Once created, each energetic LW lepton will mostly decay into a hard electron and $Z$. The electrons will have a very large $p_{T}$ because they are produced in a two body decay of a heavy 
LW lepton, and also because the LW state itself has a large $p_{T}$. For instance, for $M_{\ell}=200 \mathrm{GeV}$ the $p_{T}$ distribution of the final hardest electron is centered in $p_{T} \sim 200 \mathrm{GeV}$, whereas for $M_{\ell}=$ $400 \mathrm{GeV}$ is centered in $p_{T} \sim 900 \mathrm{GeV}$, see Fig. 3. Therefore, although the cut in the transverse momentum of the LW lepton suppresses the production cross section, at the same time, it allows us to impose hard cuts in the $p_{T}$ of the most energetic lepton, $p_{T} \gtrsim \mathscr{O}(200) \mathrm{GeV}$, with low impact in the signal. The hadronic $Z$ decay dominates, producing a jet pair for each vector. Therefore, the signal corresponding to the dominating channel is defined by a very energetic electron-positron pair and four jets, $e^{+} e^{-} j j j j$. Each pair of jets with an invariant mass corresponding to the $Z$, when considered together with the proper lepton $e^{ \pm}$, will have an invariant mass peaked around the LW-lepton mass.

The backgrounds for the signal here presented do not seem to cause further complications. However, a detailed SM background analysis should be performed to verify that the whole luminosity for the eejjjj signal data -with a suitable cut in the electrons' transverse momentum of $p_{T}(e)>200$ $\mathrm{GeV}$ - can be collected to perform an off-line analysis (this is known as an un-prescaled trigger). Afterwards, the peculiarity of this signal would need a proper vertexing algorithm to cope with the correct wrong vertex assignment.

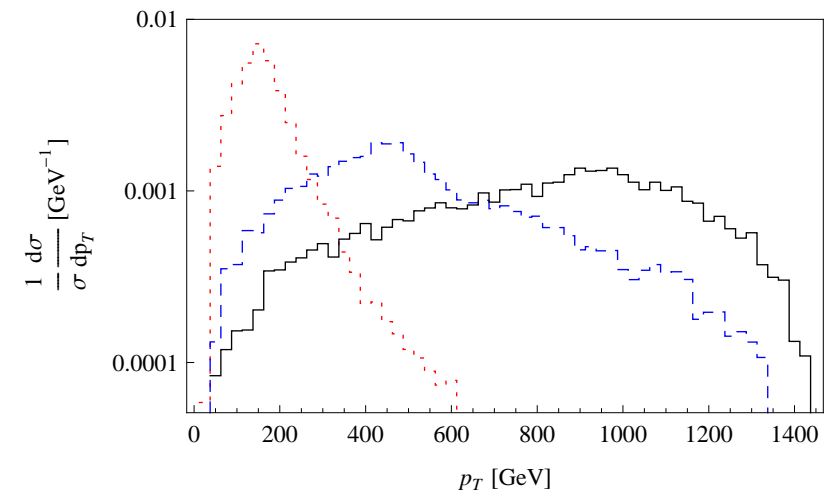

Figure $3 p_{T}$ distribution for the most energetic electron in the signal $e^{+} e^{-} j j j j$ after imposing the cut on the $p_{T}$ of the LW-leptons to obtain wrong vertex displacements greater than $\Delta x=20 \mu \mathrm{m}$. The dotted (red), dashed (blue) and black (solid) lines correspond to $\mathrm{LW}$ masses $M_{\ell}=200 \mathrm{GeV}, 300 \mathrm{GeV}$ and $400 \mathrm{GeV}$, respectively.

\section{References}

[1] B. Grinstein, D. O’Connell and M. B. Wise, Phys. Rev. D 77, 025012 (2008) [arXiv:0704.1845 [hep-ph]]; M. B. Wise, arXiv:0908.3872 [hep-ph].

[2] E. Alvarez, L. Da Rold, C. Schat and A. Szynkman, arXiv:0908.2446 [hep-ph].

[3] E. Alvarez, L. Da Rold, C. Schat and A. Szynkman, JHEP 0804026 (2008) [arXiv:0802.1061 [hep-ph]]; E. Alvarez, C. Schat, L. Da Rold and A. Szynkman, arXiv:0810.3463 [hep-ph]; C. D. Carone, Phys. Lett. B 677, 306 (2009) [arXiv:0904.2359 [hep-ph]]; T. E. J. Underwood and R. Zwicky, Phys. Rev. D 79, 035016 (2009) [arXiv:0805.3296 [hep-ph]].

[4] C. D. Carone and R. Primulando, arXiv:0908.0342 [hep-ph].

[5] F. Maltoni and T. Stelzer, JHEP 0302 (2003) 027 [arXiv:hep-ph/0208156]; J. Alwall et al., JHEP 0709 (2007) 028 [arXiv:0706.2334 [hep-ph]]. 